\title{
Effect of heparin during endovascular stroke treatment: A large single-center analysis
}

\author{
Mori F., Konda D., Morosetti D., Da Ros V., Gandini R., Koch G., Diomedi M., Sallustio F. \\ Policlinico Tor Vergata , Università degli Studi di Roma "Tor Vergata", Roma, Italy
}

Introduction: effectiveness of heparin use during endovascular mechanical thrombectomy (MT) for acute ischemic stroke (AIS) has been barely investigated. Use of i.v. heparin during arterial catheterization is largely employed (1) to prevent thrombus formation over catheters, distal embolization, clot extension and arterial reocclusion. On the other hand, anticoagulation in the early phases of acute ischemic stroke is not recommended due to the risk of serious intracranial hemorrhage (ICH) (2).

Aim: to assess effectiveness and safety of heparin use during MT for AIS in a real-world setting.

\section{Methods:}

Patients

361 patients with anterior circulation AIS undergoing MT were retrospectively analyzed from our stroke registry. Based on intra-procedural use of heparin, patients were grouped in heparin (H+) and no-heparin (H-).

Outcomes

The effect of heparin use on MT effectiveness was tested by comparing rates of successful reperfusion defined as a TICI score $\geq 2 b$ ( 3 ) between H+ and H- groups. Effect of heparin use on safety was tested by comparing rates of hemorrhagic complications defined according to the ECASS criteria (no hemorrhage, hemorrhagic infarction-1, hemorrhagic infarction-2, parenchymal hematoma-1, parenchymal hematoma-2) (4), and symptomatic ICH (sICH) defined as an hemorrhage associated with an increase of at least 4 points in the NIHSS.

Age, gender, NIHSS, site of occlusion, history of hypertension, diabetes, smoking, atrial fibrillation, ASPECTS on non-contrast CT (5), collaterals adequacy on pretreatment CT-Angiography (6), intravenous thrombolisys (IVT), general anesthesia, type of device for thrombectomy, procedural times were also compared between groups to control for an effect of possible confounding factors.

Results:

Table 1. Demographics and baseline characteristics

\begin{tabular}{|c|c|c|c|}
\hline & No Heparin $(n=161)$ & Heparin $(n=200)$ & $\mathrm{p}$ \\
\hline Age, years (mean $\pm S D)$ & $74 \pm 14$ & $68.9 \pm 12.2$ & 0.001 \\
\hline Gender (male) (\%) & $62(38.5)$ & $85(42.5)$ & 0.45 \\
\hline Pre-stroke mRS 0-2 (\%) & $156(96.8)$ & 198(99) & 0.24 \\
\hline Hypertension (\%) & 115(71.4) & 147(73.5) & 0.72 \\
\hline Diabetes (\%) & $24(14.9)$ & $39(19.5)$ & 0.26 \\
\hline Atrial Fibrillation (\%) & 72(44.7) & 95(47.5) & 0.67 \\
\hline Current Smoking (\%) & $25(15.5)$ & $32(16)$ & 1.00 \\
\hline Glycemia (mean $\pm S D$ ) & $128.2 \pm 42.8$ & $134.1 \pm 50.7$ & 0.36 \\
\hline NIHSS (mean $\pm S D$;median(range)) & $17.1 \pm 5.01 ; 18(2-26)$ & $18.1 \pm 4.1 ; 19(3-25)$ & 0.17 \\
\hline Systolic BP (mean \pm SD) & $144.9 \pm 26.4$ & $148.3 \pm 26.8$ & 0.47 \\
\hline Diastolic BP (mean \pm SD) & $79 \pm 16.8$ & $82.3 \pm 14.8$ & 0.07 \\
\hline ASPECTS (mean $\pm S D$; median(range)) & $8 \pm 1.6 ; 8(2-10)$ & $7.4 \pm 2.1 ; 8(2-10)$ & 0.009 \\
\hline MCA occlusion (\%) & 102(63.3) & $123(61.5)$ & 0.74 \\
\hline Terminal ICA (\%) & $4(2.4)$ & 2(1) & 0.41 \\
\hline T occlusion (\%) & $9(5.5)$ & $7(3.5)$ & 0.44 \\
\hline Tandem occlusion (\%) & $46(28.5)$ & $68(34)$ & 0.3 \\
\hline Good collaterals $(\%)$ & $99(61.4)$ & $128(64)$ & 0.66 \\
\hline
\end{tabular}

NIHSS= National Institute of Health Stroke Scale; BP: blood pressure; ASPECTS= Alberta Stroke Program Early CT Score; $M C A=$ middle cerebral artery; ICA = internal carotid artery; $T$ occlusion= combined occlusion of terminal ICA, MCA and ACA

\section{Table 3. Safety and efficacy outcomes}

\begin{tabular}{lccc|ccc|ccc}
\hline & & Overall & & \multicolumn{3}{c|}{$\mathrm{MT}$} & \multicolumn{3}{c}{ IV+MT } \\
& $\mathrm{H}-(\mathrm{n}=161)$ & $\mathrm{H}+(\mathrm{n}=200)$ & $\mathrm{p}$ & $\mathrm{H}-(\mathrm{n}=49)$ & $\mathrm{H}+(\mathrm{n}=94)$ & $\mathrm{p}$ & $\mathrm{H}-(\mathrm{n}=112)$ & $\mathrm{H}+(\mathrm{n}=106)$ & $\mathrm{p}$ \\
Successful reperfusion (\%) & $133(82.6)$ & $139(69.5)$ & 0.004 & $41(83.6)$ & $61(64.8)$ & 0.02 & $92(82.1)$ & $78(73.5)$ & 0.14 \\
24h ASPECTS (median;range) & $6(0-10)$ & $4(0-10)$ & $<0.001$ & $6(0-10)$ & $5(0-10)$ & 0.03 & $6(0-10)$ & $5(0-10)$ & $<0.001$ \\
Any ICH (\%) & $58(36)$ & $74(37)$ & 0.9 & $19(38.7)$ & $34(36.1)$ & 0.85 & $39(34.8)$ & $40(37.7)$ & 0.67 \\
SAH & $3(1.8)$ & $2(1)$ & 0.66 & $1(2)$ & $1(1)$ & 1.00 & $2(1.7)$ & $2(1.8)$ & 1.00 \\
HI-1 & $3(1.8)$ & $7(3.5)$ & 0.52 & $2(4)$ & $3(3.1)$ & 1.00 & $1(0.8)$ & $4(3.7)$ & 0.2 \\
HI-2 & $12(7.4)$ & $10(5)$ & 0.37 & $7(14.2)$ & $5(5.3)$ & 0.1 & $5(4.4)$ & $5(4.7)$ & 1.00 \\
PH-1 & $15(9.3)$ & $23(11.5)$ & 0.6 & $2(4)$ & $9(9.5)$ & 0.33 & $13(11.6)$ & $12(11.3)$ & 1.00 \\
PH-2 & $25(15.5)$ & $32(16)$ & 1.00 & $7(14.2)$ & $14(14.8)$ & 1.00 & $18(16)$ & $14(13.2)$ & 0.57 \\
Symptomatic ICH (\%) & $16(9.9)$ & $19(9.5)$ & 1.00 & $3(6.1)$ & $12(12.7)$ & 0.26 & $7(6.2)$ & $14(13.2)$ & 0.1 \\
3-month mRS 2 (\%) & $66(40.9)$ & $73(36.5)$ & 0.38 & $13(26.5)$ & $31(32.9)$ & 0.45 & $53(47.3)$ & $41(38.6)$ & 0.21 \\
3-month mRS 3 (\%) & $74(45.9)$ & $91(45.5)$ & 1.00 & $14(28.5)$ & $41(43.6)$ & 0.1 & $60(53.5)$ & $49(46.2)$ & 0.34 \\
All-cause mortality (\%) & $49(30.4)$ & $63(31.5)$ & 0.9 & $18(36.7)$ & $35(37.2)$ & 1.00 & $31(27.6)$ & $26(24.5)$ & 0.64
\end{tabular}

$M T=$ mechanical thrombectomy; $I V+M T=$ intravenous thrombolysis plus mechanical thrombectomy; ASPECTS=Alberta Stroke Program Early CT Score; $I \mathrm{CH}=$ Intracranial haemorrhage; $\mathrm{SAH}=$ Subarachnoid haemorrhage; $\mathrm{HI}=$ Hemorrhagic Infarction; $\mathrm{PH}=\mathrm{Parenchymal}$ haematoma; $m R S=$ modified Rankin Scale.

\begin{tabular}{|ccccc|}
\multicolumn{4}{c}{ Table 4. Multivariable regression model: best predictors of successful reperfusion } \\
\hline Variables & OR & SE & $\mathbf{9 5 \%} \mathbf{C l}$ & $\boldsymbol{P}$ \\
\hline Stentriever vs Aspiration & 0.45 & 0.15 & $0.22-0.89$ & 0.02 \\
\hline CT ASPECTS & 1.2 & 0.08 & $1.05-1.37$ & 0.007 \\
\hline Heparin & 0.49 & 0.17 & $0.25-0.97$ & 0.04
\end{tabular}

OR: Odds Ratio; SE: standard error; Cl: confidence interval

Our study reveals that heparin use during endovascular procedure of MT for anterior circulation AIS is safe and does not improve clinical outcome but is associated with lower chance of reperfusion. 